\title{
Procesos para la obtención de hilo de fibra de llama (Llama glama) chaku y evaluación de características
} físicas

\section{Processes for the obtaining of thread of the flame fiber (Llama glama) chaku and evaluation of physical characteristics}

Maritza Lucia Vaca Cardenas. ${ }^{1}$, Wendy Gabriela Chafla Cando. ${ }^{2}$, Elsa Margarita Remache Sisa. ${ }^{3} \&$ Luis Agustin Condolo Ortiz. ${ }^{4}$

Recibido: 19-04-2019 / Revisado: 25-05-2019 /Aceptado: 28-06-2019/ Publicado: 15-07-2019

\begin{abstract}
.
DOI: https://doi.org/10.33262/cienciadigital.v3i3.1.707

In the ESPOCH the performance in the flame fiber was evaluated, in the laboratory tests 4 fiber characteristics were evaluated: fiber length, number of curls, spin resistance and tissue resistance, for which 50 fiber samples were taken Thick and fine to determine the length and number of curls; for the yarn resistance, 10 samples were used and 1 sample of fine and coarse fiber was used for the strength of the fabric. The tests were analyzed in the tannery laboratory of the FCP-ESPOCH, the statistical analysis that was applied was the T-student test, by which different physical parameters were evaluated. The results indicate that the fine fiber presented an average length of $17 \pm 9$ $\mathrm{cm}$, with a coefficient of variability of $(0.46 \%)$ while the coarse fiber presented an average length of $(24.2 \pm 2 \mathrm{~cm})$, with a coefficient of variability of $(13.85 \%)$. As for
\end{abstract}

\footnotetext{
1 Escuela Superior Politécnica de Chimborazo, Facultad de Ciencias Pecuarias. Riobamba, Ecuador. maritza.vaca@espoch.edu.ec

2 Escuela Superior Politécnica de Chimborazo, Facultad de Ciencias Pecuarias. Riobamba, Ecuador. gabychafla98@hotmail.es

3 Escuela Superior Politécnica de Chimborazo, Facultad de Ciencias Pecuarias. Riobamba, Ecuador. elsamargremaches@gmail.com

4 Escuela Superior Politécnica de Chimborazo, Facultad de Ciencias Pecuarias. Riobamba, Ecuador. luis.condolo@espoch.edu.ec
} 
the average number of curls per centimeter in the fine fiber, it was $2.78 \pm 0.92$ curls / $\mathrm{cm}$, with a coefficient of variability of $(0.01 \%)$ while the coarse fiber showed an average of $7 \pm 0.07$ curls / $\mathrm{cm}$, with a coefficient of variability of $(0.56 \%)$. The average resistance of fine fiber was $9.7 \pm 7.6 \mathrm{lb}$, and there was no variability, while coarse fiber presented an average of $15 \pm 9 \mathrm{lb}$, with a coefficient of variability of $(1.1 \%)$. The results showed highly significant differences $(\mathrm{p} \leq 0,01)$ in the number of curls, but in the resistance no significant differences were found $(\mathrm{p} \geq 0,05)$ in comparison of the fine and coarse fiber. In addition, sensory tests of color of the fiber were carried out, establishing the nut color for the two fibers and with respect to the softness, significant differences were established

Keywords: Panties, shearing, fleece, fine fiber and coarse.

\section{Resumen.}

En la ESPOCH se evaluó el rendimiento en la fibra de llama, en las pruebas laboratorio se valoraron 4 características de la fibra: longitud de fibra, numero de rizos, resistencia de hilado y resistencia del tejido, para lo cual se tomaron 50 muestras de fibra gruesa y fina para determinar la longitud y numero de rizos; para la resistencia del hilado se utilizó 10 muestras y para resistencia del tejido se utilizó 1 muestra de fibra fina y gruesa. Las pruebas se analizaron en el laboratorio de curtiembre de la FCP-ESPOCH, el análisis estadístico que se aplico fue la prueba de T-student, mediante el cual se evaluaron diferentes parámetros físicos. Los resultados indican que la fibra fina presentó una longitud promedio de $17 \pm 9 \mathrm{~cm}$, con un coeficiente de variabilidad de $(0,46 \%)$ mientras que la fibra gruesa presento una longitud promedio de $(24.2 \pm 2 \mathrm{~cm})$, con un coeficiente de variabilidad de $(13,85 \%)$. En cuanto al número promedio de rizos por centímetros en la fibra fina fue de 2,78 $\pm 0,92 \mathrm{rizos} / \mathrm{cm}$, con un coeficiente de variabilidad de $(0,01 \%)$ mientras que la fibra gruesa presento un promedio de $7 \pm 0.07$ rizos/cm, con un coeficiente de variabilidad de $(0,56 \%)$. La resistencia promedio que presento la fibra fina fue de $9,7 \pm 7,6 \mathrm{lb}$, y no presento variabilidad, mientras que la fibra gruesa presentó un promedio de $15 \pm 9 \mathrm{lb}$, con un coeficiente de variabilidad de (1.1 $\%)$. Los resultados mostraron diferencias altamente significativas $(p \leq 0,01)$ en el número de rizos, pero en la resistencia no se encontraron diferencias significativas 
$(\mathrm{p} \geq 0,05)$ en comparación de la fibra fina y gruesa. Además, se realizó pruebas sensoriales de color de la fibra estableciendo el color nuez para las dos fibras y con respecto a la suavidad se estableció diferencias significativas.

Palabras claves: Bragas, esquila, vellón, fibra fina y fibra gruesa.

\section{Introducción.}

Los camélidos sudamericanos (CSA), constituyen un recurso genético de gran importancia social, económica, cultural y científica para el Perú y algunos de los países de la Región Andina. Son una fuente de riqueza pecuaria y genética importante de las poblaciones andinas, dentro de ellos la llama por su fibra de gran valor (Quispe, 2014).

La llama (Lama glama) es el camélido doméstico más grande y se encuentra adaptada a un amplio rango de condiciones medioambientales, siendo de gran importancia para los ecosistemas de los Andes, por sus múltiples posibilidades de uso como productor de fibra y carne, su empleo en el transporte de carga y el múltiple uso del estiércol, así como por su importancia cultural (Huarcaya, 2015)

En la Estación Experimental de Tunshi de la ESPOCH, existe una área de manejo de animales, en la cual encontramos a las llamas, que son manejadas bajo pastoreo y en corrales, en la actualidad, las llamas están distribuidas casi en todos los continentes, excepto Asia; sin embargo, los países de Bolivia y Perú resaltan con la mayor población a nivel mundial. En el Perú, el departamento de mayor población de llamas es la región Puno con 435,120 llamas que representa el $35.48 \%$ a nivel del país (INEI, 2015). Mientras que en el Ecuador la provincia de Bolívar es mayor productora de llamas, cuenta con un promedio de 2750 animales.

Siguayro R. (2009) afirma que las llamas poseen dos capas de fibra; una capa externa la cual presenta fibras muy gruesas y largas mientras que la capa interna presenta fibras cortas de extrema fineza y suavidad, esta fracción se puede ganar a través del proceso de descerdado. Quispe J. (2012) asumen que los compradores y productores de fibra coinciden en que existe una demanda de fibra de llama pero por razones de los bajos índices de extracción, fluctuación de la calidad y cantidad no se aprovecha este potencial. 
Un problema adicional, se origina a la hora de darse la esquila de los animales, donde los acopiadores pagan precios irrisorios por el kilo de fibra (Vedia, J. 2007).

La fibra de llama es utilizada para la fabricación de diversos artículos como es el caso de los artículos de adorno, aunque se lo utiliza más para la vestimenta, casi en la mayoría de los casos se utiliza al máximo la fibra para evitar pérdidas para el productor-cliente.

El presente estudio permite conocer las características físicas de la fibra de llama chaku procedente de la estación experimental Tunshi, de igual forma conocer los procesos desde la esquila hasta la obtención del hilo para la fabricación de vestimenta.

\section{Metodologia.}

\section{Actividades de campo}

La investigación se llevó a cabo en el Estación Experimental Tunshi de la ESPOCH ubicada en el kilómetro 12 vía a Licto y con una extensión actual de 60 hectáreas, cuenta con el área pecuaria mismo que tiene como objetivo la producción y explotación pecuaria. La esquila se realizó en el mes de marzo para la cual primero se seleccionó a una llama de la raza chaku, posteriormente se llevó a cabo la limpieza del vellón del animal, luego se procedió al derribe del animal sobre la una manta teniendo cuidado con el cuello del mismo, se cubrió los ojos del animal para evitar que el entorno lo estresara, se sujetó las extremidades con soguillas y se inmovilizó hacia las estacas seguidamente se procedió a la esquila manual del animal para lo cual se utilizó una tijera con la cual se procedió a esquilar desde el vientre hasta el lomo, después se realizó el esquilado de las bragas, una vez que se obtuvo el vellón del animal se procedió a retirar las impurezas y a clasificar la fibra bragas y manto.

Una vez realizado el envellonado de la fibra se llevó a cabo el pesado del vellón completo para la cual se utilizó una balanza dando un peso de 2,5 lb, mientras que solo el peso del manto fue de $1 \mathrm{lb}$ y las bragas tuvo un peso de $1,3 \mathrm{lb}$ libras y $0.2 \mathrm{lb}$ la basura o tierra presente en la fibra, seguidamente se utilizó una olla con agua caliente en la cual se sumergió la fibra ya que presentaba liendres a continuación colocamos detergente como es la deja en la fibra y se lavó bien con agua fría hasta quitar todo el detergente y se procedió a colocar la fibra en un tendedero para el secado, una vez que se encontraba seco se realizó el deserdado y el escarmenado de la fibra. 
Cuadro 1. Pesos realizados a la fibra fina y gruesa

\begin{tabular}{|l|l|l|l|}
\hline \multicolumn{2}{|c|}{ Fibra sucia } & \multicolumn{2}{c|}{ Fibra lavada } \\
\hline DATOS & PESOS & DATOS & PESOS \\
\hline Peso del vellón & $2.5 \mathrm{lb}$ & Fibra fina & 0.6 \\
\hline fibra fina & $11 \mathrm{~b}$ & Fibra gruesa & 0,9 \\
\hline Fibra gruesa & $1.3 \mathrm{lb}$ & Cerda & 0.5 \\
\hline Basura o tierra & $0.2 \mathrm{lb}$ & & \\
\hline
\end{tabular}

Fuente: grupo investigador

Rendimiento del vellón

$$
\begin{gathered}
R=\frac{W \text { fibra limpia }}{W \text { fibra sucia }} \times 100 \\
R=\frac{1 l b}{2.5 l b} \times 100 \\
R=40 \%
\end{gathered}
$$

Posteriormente se efectuó al hilado manual para esto primero se realizó el guango seguidamente se empezó a hilar en el huso el mismo que lleva en la parte inferior una pieza llamada tortero aquí es necesario llenar dos husos, después de eso se procedió a hacer madeja y seguidamente a realizar el del torcido del hilo a lado izquierdo para nuevamente hacer la madeja la cual se encuentra listo para empezar a realizar el tejido. Por último se ejecutó el tejido de una bufanda con la fibra fina y con la fibra gruesa se realizó 2 tapetes para lo cual se utilizaron agujones $\mathrm{N}^{\circ} 3$ y crushed.

\section{Pruebas físicas del laboratorio}

Las pruebas físicas se realizaron en el laboratorio de curtiembre de la FCP para dichas pruebas físicas como longitud de fibra, numero de rizos, se utilizó una regla, pinzas y cartulina y para la resistencia del hilado y resistencia del tejido se utilizó el elastómero y el 
lastometro, estas pruebas se realizaron con 10 muestras de fibra fina y fibra gruesa de la llama chaku.

\section{Pruebas de Análisis sensorial}

Se realizó pruebas de color y de suavidad al tacto del tejido de la fibra de llama, el mismo que se realizó en las aulas de la FCP, utilizando nuestros órganos de los sentidos (vista y del tacto), en la segunda prueba se aplicó la prueba de ordenamiento.

\section{Resultados.}

\section{Longitud de fibra}

Cuadro2. Longitud de fibra fina y gruesa de la llama chaku.

\begin{tabular}{|l|r|r|}
\hline DATOS & FIBRA FINA & FIBRA GRUESA \\
\hline Promedio $(\mathrm{cm})$ & $11,51 \mathbf{a}$ & $18,33 \mathbf{b}$ \\
\hline Máximo & 17 & 24,2 \\
\hline Mínimo & 9 & 2 \\
\hline Varianza & 2,30 & 15,93 \\
\hline Desviación estándar & 5,31 & 253,84 \\
\hline Coeficiente de variación & 0,46 & 1,85 \\
\hline
\end{tabular}

Fuente: Elaboración propia

Letras desiguales indican que existen diferencias altamente significativas $(p \leq 0.01)$. El Cuadro 2, muestran valores promedio de longitud de fibra fina y gruesa de llama Chaku. La longitud promedio encontrado en la fibra fina de la llama Chaku fue de $17 \pm 9 \mathrm{~cm}$, con un coeficiente de variabilidad de 0,46\% y en la fibra gruesa fue de $24,2 \pm 2 \mathrm{~cm}$, con un coeficiente de variabilidad de $1,85 \%$. Estos valores promedio de longitud de fibra mostraron diferencias altamente significativas $(\mathrm{p} \leq 0,01)$ entre el tipo de fibra. (Sierra, 2014) Menciona que el promedio de longitud de la fibra de llama debe encontrarse entre 10,44 cm. Y 15,25 $\mathrm{cm}$, pero estos datos pueden variar ya sea por la edad del animal o el tiempo de esquila que se realiza. 


\section{Numero de rizos}

Cuadro 3. Numero de rizos de fibra fina y gruesa de la llama chaku.

\begin{tabular}{|l|r|r|}
\hline DATOS & $\begin{array}{c}\text { FIBRA } \\
\text { FINA }\end{array}$ & $\begin{array}{c}\text { FIBRA } \\
\text { GRUESA }\end{array}$ \\
\hline Promedio $(\mathrm{cm})$ & $1,96 \mathbf{a}$ & $1,14 \mathbf{b}$ \\
\hline Máximo & 2,78 & 7,00 \\
\hline Mínimo & 0,92 & 0,07 \\
\hline Varianza & 0,13 & 0,80 \\
\hline Desviación estándar & 0,02 & 0,64 \\
\hline Coeficiente de variación & 0,01 & 0,56 \\
\hline
\end{tabular}

\section{Fuente: Elaboración propi}

Letras desiguales indican que existen diferencias altamente significativas $(p \leq 0.01)$ En el Cuadro 3, se presenta un resumen de los valores promedio del número de rizos por centímetro de fibra fina y gruesa de la llama Chaku. El número promedio de rizos por centímetros en fibra fina de llama Chaku fue de 2,78 $\pm 0,9$ rizos $/ \mathrm{cm}$, con un coeficiente de variabilidad de $0,01 \%$ y en fibra gruesa fue de $7 \pm 0,7 \mathrm{rizos} / \mathrm{cm}$, con un coeficiente de variabilidad de 0,56 $\%$; Comparando los valores promedio del número de rizos por centímetro, se encontraron diferencias altamente significativas $(\mathrm{p} \leq 0,01)$ entre los dos tipos de fibras. Estos resultados reafirman lo manifestado por (Aliaga, 2006) manifiesta que frecuencia de rizos en la mecha siempre se asocia a fibras de buena calidad, de manera que las fibras rizadas tienen mayores cualidades textiles que las no rizadas por lo tanto deben estarse entre 2,33 $\pm 2,2$ rizos $/ \mathrm{cm}$ lo que indica que la fibra de la llama no es de buena calidad ya que el número de rizos es menor al número mencionado, esto se debe a que la fibra estaba con presencia de liendres lo cual bajo su calidad.

\section{Resistencia de la fibra}

Cuadro 4. Resistencia del hilado de fibra la fina y gruesa de la llama chaku.

\begin{tabular}{|l|c|c|}
\hline DATOS & FIBRA FINA & FIBRA GRUESA \\
\hline Promedio $(\mathrm{cm})$ & $8,93 \mathbf{a}$ & $12,58^{\mathrm{a}}$ \\
\hline
\end{tabular}




\begin{tabular}{|l|l|l|}
\hline Máximo & 9,70 & 15,00 \\
\hline Mínimo & 7,60 & 9,00 \\
\hline Varianza & 0,13 & 2,36 \\
\hline Desviación estándar & 0,02 & 5,55 \\
\hline Coeficiente de variación & 0,00 & 1,11 \\
\hline
\end{tabular}

Fuente: Elaboración propia

Letras iguales indican que no existen diferencias estadísticas significativas $(p \geq 0.05)$. En el Cuadro 4, se presenta un resumen de los valores promedio de resistencia de fibra fina y gruesa de la llama Chaku. La resistencia promedio en fibra fina de llama Chaku fue de 9,70 \pm 7,60 lb, con un coeficiente de variabilidad de $0 \%$ y en fibra gruesa fue de 15,00 $\pm 9,00 \mathrm{lb}$ con un coeficiente de variabilidad de 1,1\%. Estos valores promedio de resistencia de fibra mostraron que no existen diferencias significativas $(p \geq 0,05)$ lo que significa que la fibra fina y gruesa resisten por igual.

\section{Pruebas de resistencia del tejido}

Cuadro 5. Resistencia del tejido con fibra fina y gruesa de llama

\begin{tabular}{|c|c|c|c|c|c|}
\hline \multicolumn{3}{|c|}{ TEJIDO CON EL HILO FINO } & \multicolumn{3}{c|}{ TEJIDO CON EL HILO GRUESO } \\
\hline Segundos & Bares & PSI & Segundos & Bares & PSI \\
\hline $\mathbf{2 . 3 9}$ & 1.9 & 29 & 3,04 & 2,13 & 36 \\
\hline
\end{tabular}

Fuente: Elaboración propia

En la prueba de resistencia del tejido se realizó en el lastometro por la cual se determinó que el tejido realizado con el hilo grueso resistió por más tiempo (3.04s), esto pudo ocurrir debido al mayor grosos que presentaba el hilo así también pudo afectar el tipo de tejido que se realizó, a diferencia del tejido con el hilo fino $(2,39 \mathrm{~s})$.

\section{Pruebas de color}

Cuadro 6. Pruebas de color de la fibra fina y gruesa de la llama chaku

\begin{tabular}{|c|c|c|c|}
\hline $\begin{array}{c}\text { Fibra Fina } \\
\text { (color) }\end{array}$ & $\%$ & $\begin{array}{c}\text { Fibra Guesa } \\
\text { (color) }\end{array}$ & $\%$ \\
\hline
\end{tabular}




\begin{tabular}{|l|l|l|r|}
\hline Azclime & 20 & Azclime & 30 \\
\hline Nuez & 60 & Nuez & 60 \\
\hline Beige & 10 & Chocolate & 10 \\
\hline Chocolate & 10 & \multicolumn{2}{|c}{} \\
\hline
\end{tabular}

Fuente: Elaboración propia

En el cuadro 6, se presentan los resultados del análisis de color de la fibra de llama chaku diferenciando la fibra fina de la gruesa, en el primero se distinguió 4 colores en la cual resalta el color nuez con un $60 \%$, en la fibra gruesa se obtuvo un $60 \%$ en el color nuez, por la cual no se distinguió diferencias de tonos de colores en cuanto a la fibra de llama.

\section{Pruebas de suavidad al tacto}

Cuadro 7. Pruebas de suavidad al tacto de la fibra fina y gruesa de llama chaku

\begin{tabular}{|l|}
\hline $\begin{array}{l}\text { RESULTADOS } \\
\text { OBTENIDOS }\end{array}$ \\
\hline$A=$ CONEJO \\
\hline$B=$ ALGODÓN \\
\hline C $=$ ALPACA \\
\hline$D=$ LLAMA FINA \\
\hline$E=$ LLAMA GRUESA \\
\hline$G=$ OVEJA GRUESA \\
\hline$F=$ OVEJA FINA \\
\hline
\end{tabular}

\begin{tabular}{|l|}
\hline $\begin{array}{l}\text { RESPUESTAS } \\
\text { CORRECTAS }\end{array}$ \\
\hline 1. CONEJO A \\
\hline 2. ALGODO B \\
\hline 3. ALPACA C \\
\hline 4. LLAMA FINA D \\
\hline 5. LLAMA GRUESA E \\
\hline 6. OVEJA FINA F \\
\hline 7. OVEJA GRUESA G \\
\hline
\end{tabular}

Fuente: Elaboración propia

Prueba de chi - cuadrado experimental y prueba de DMS

$X^{2} \exp =21.7$

$X^{2} \mathrm{tab}=10.64$

$X^{2} \exp >X^{2} t a b$

$\mathrm{DMS}=13.25$

En el cuadro 7 se observa que Para la suavidad al tacto se aplicó pruebas discriminativas, denominada prueba de ordenamiento, en la cual se aplicó la fórmula establecida de chi -cuadrado experimental, obteniendo un resultado de 21.7 en 
comparación con el chi - cuadro tabular (10.64), el primero es mayor al segundo dato, en si se rechaza la hipótesis nula ya que existen diferencias significativas, por lo tanto se aplica la fórmula del DMS obteniendo un resultado de 13.25, conociendo así el ordenamiento de las fibras de mayor a menor suavidad (A,B,C,D,E,G,F).

\section{Conclusiones.}

- El rendimiento de la fibra de llama chaku, no fue rentable debido a que el animal no contaba con un cuidado especial, y sobre todo no se realizó la esquila en el tiempo adecuado (condiciones ambientales) lo cual bajo su calidad.

- La resistencia del hilado no difiere con el tipo de fibra que se realice el hilado ya que no presentan diferencias significativas.

- Las características físicas como longitud y número de rizos de la fibra de llama chaku se encuentran dentro de los rangos que establecen otros autores.

\section{Referencias bibliográficas.}

- Aliaga. (2006). Parametros fenotipicos de algunas caracteristicas fisicas de la fibra de llamas Cha'ccu. Huancayo - Perú: UNCP.

- Huarcaya, F. d. (2015). Características Tecnológicas de la Fibra de Llama (Lama glama). Peru.

- [INEI] Instituto Nacional de Estadistica e Informatica. 2012. Base de datos del IV Censo Nacional Agropecuario. [Internet]. Disponible en: http://censos.inei.gob.pe/cenagro/tabulados/

- Mamani, W, Calsin, B, Quispe J. 2012. Diametro de fibra y pelos de llamas K'ara y Ch'acu del CIP La Raya, UNA--Puno. Rev ALLPAKA 16: 51-57

- Pinares, R., L. Chipa, R. Paúcar, \& E. Quispe, (2014). Estudio de la diferencia post y pre descerdado de cinco características textiles de la fibra de llama (Lama glama) Chaku. Revista de Investigacion en Ciencias Sociales y Tecnologicas. Universidad Nacional Micaela Bastidas de Apurimac Vol 1 pg 69-77 
- Sierra, J. (2014). Características fisicas de la fibra de llamas. Obtenido de http://repositorio.uncp.edu.pe/bitstream/handle/UNCP/1836/Tesis\%20Sierra.pdf?se quence $=1 \&$ isAllowed $=\mathrm{y}$

- Vedia, J. (2007). Estudio de la fibra de llama (vellón) según ecotipos en la provincia Sajama del departamento de Oruro. Tesis. Tarija, Bolivia. UJNS. 86 p. 


\section{PARA CITAR EL ARTÍCULO INDEXADO.}

Vaca Cardenas, M., Chafla Cando, W., Remache Sisa, E., \& Condolo Ortiz, L. (2019). Procesos para la obtención de hilo de fibra de llama (Llama glama) chaku y evaluación de características físicas Procesos para la obtención de hilo de fibra de llama (Llama glama) chaku y evaluación de características físicas. Ciencia Digital, 3(3.1), 341-352. https://doi.org/10.33262/cienciadigital.v3i3.1.707

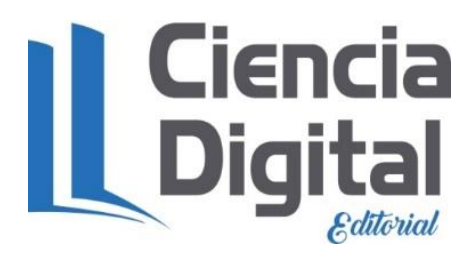

El artículo que se publica es de exclusiva responsabilidad de los autores y no necesariamente reflejan el pensamiento de la Revista Ciencia Digital.

El artículo queda en propiedad de la revista y, por tanto, su publicación parcial y/o total en otro medio tiene que ser autorizado por el director de la Revista Ciencia Digital.
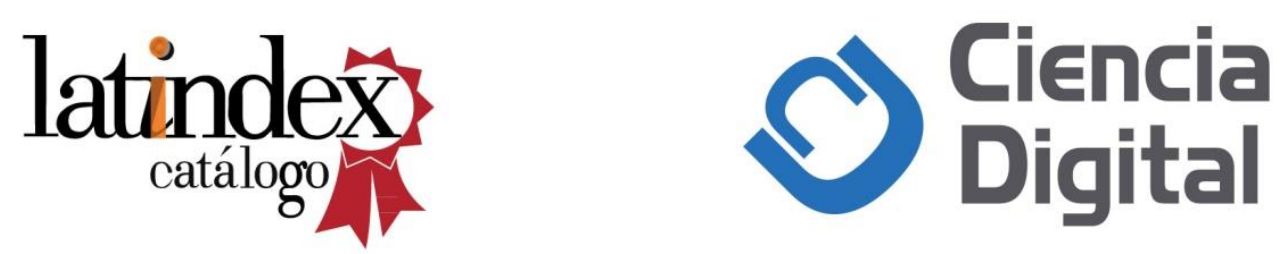